J. Inst. Math. Jussieu (2022), 21(6), 2247-2251

doi:10.1017/S1474748020000705 C The Author(s), 2021. Published by Cambridge University 2247 Press. This is an Open Access article, distributed under the terms of the Creative Commons

Attribution licence (http://creativecommons.org/licenses/by/4.0/), which permits unrestricted re-use, distribution, and reproduction in any medium, provided the original work is properly cited.

\title{
ERRATUM
}

\section{PERIODS OF DRINFELD MODULES AND LOCAL SHTUKAS WITH COMPLEX MULTIPLICATION - ERRATUM}

\author{
URS HARTL (D) ${ }^{1}$ AND RAJNEESH KUMAR SINGH ${ }^{2}$ \\ ${ }^{1}$ Universität Münster, Mathematisches Institut, Einsteinstr. 62, \\ D-48149 Münster, Germany (https://www.uni-muenster.de/Arithm/hartl/) \\ ${ }^{2}$ Ramakrishna Mission, Vivekananda University, PO Belur Math, Dist Howrah \\ 711202, West Bengal, India
}

(Received 8 June 2020; revised 11 November 2020; accepted 20 November 2020; first published online 12 February 2021)

\section{B. Errata}

\section{B.1. First Error}

In [2, formulas (1.13) and (1.12) and Definition 5.21] it is claimed that $v\left(\omega_{\psi}\right)$ and $v_{\psi}(u)$ are integers. However, in general they only lie in the rational numbers $\mathbb{Q}$, because the valuation $v$ is normalized to be an isomorphism $v: Q_{v}^{\times} / A_{v}^{\times} \stackrel{\sim}{\longrightarrow}$, but the arguments of $v$

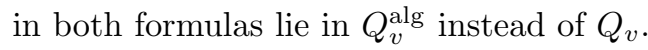

This error is harmless, as the integrality of $v_{\psi}(u)$ and $v\left(\omega_{\psi}\right)$ is nowhere used.

\section{B.2. Second Error}

In [2, formula (1.13) and Definition 4.10] there is an error in the definition of $v\left(\omega_{\psi}\right)$.

As in most of [2], we fix a finite separable semi-simple $Q$-algebra $E$. That is, $E$ is a product of finite separable field extensions of $Q$. We fix a finite place $v$ of $Q$ and consider the decomposition of the separable $Q_{v}$-algebra $E_{v}:=E \otimes_{Q} Q_{v}=E_{v, 1} \times \cdots \times E_{v, s}$ into a product of finite field extensions $E_{v, i}$ of $Q_{v}$ as after [2, Definition 4.1]. We fix a finite Galois extension $K \subset Q^{\text {alg }}$ of $Q$ and we let $L:=K_{v} \subset Q_{v}^{\text {alg }}$ be the closure of $K$. It is a finite Galois extension of $Q_{v}$. We fix a $\psi \in H_{E}$. The canonical extension $\psi \otimes \operatorname{id}_{Q_{v}}: E_{v} \rightarrow$ $L$ will be denoted again by $\psi$ and factors through the quotient $E_{v, i(\psi)}$ of $E_{v}$; see [2, Definition 4.5].

Let $\underline{\hat{M}}$ be a local shtuka over $R:=\mathcal{O}_{L}$ with complex multiplication by $\mathcal{O}_{E_{v}}$ as in $[2$, Definition 4.3]. It may arise from a good model $\underline{\mathcal{M}}$ of an $A$-motive over $R$ as in [2,

Both authors acknowledge support by the Deutsche Forschungsgemeinschaft (DFG) in the form of SFB 878 and Germany's Excellence Strategy EXC 2044-390685587 "Mathematics Münster: DynamicsGeometry-Structure". The first author was also supported by the DFG in form of Project-ID 427320536 SFB 1442. 
Example 3.2]. We consider the one-dimensional $L$-vector space

$$
\begin{aligned}
\mathrm{H}^{\psi}(\underline{\hat{M}}, L) & :=\left\{\omega \in \mathrm{H}_{\mathrm{dR}}^{1}(\underline{\hat{M}}, L):[a]^{*} \omega=\psi(a) \cdot \omega \forall a \in \mathcal{O}_{E_{v}}\right\} \\
& \stackrel{\sim}{\longrightarrow} \mathrm{H}_{\mathrm{dR}}^{1}(\underline{\hat{M}}, L) /\left([a]^{*}-\psi(a): a \in \mathcal{O}_{E_{v}}\right) \cdot \mathrm{H}_{\mathrm{dR}}^{1}(\underline{\hat{M}}, L),
\end{aligned}
$$

where the isomorphism comes from [2, Proposition 4.9] using the fact that $E$ is separable over $Q$.

In [2, formula (1.13) and Definition 4.10] there is an error in the definition of $v\left(\omega_{\psi}\right)$ for $L \llbracket y_{i(\psi)}-\psi\left(y_{i(\psi)}\right) \rrbracket$-generators $\omega_{\psi}$ of $\mathrm{H}^{\psi}\left(\underline{\hat{M}}, L \llbracket y_{i(\psi)}-\psi\left(y_{i(\psi)}\right) \rrbracket\right)$. Namely, there, as a reference integral structure on the $L$-vector space

$$
\mathrm{H}^{\psi}(\underline{\hat{M}}, L)=\mathrm{H}^{\psi}\left(\underline{\hat{M}}, L \llbracket y_{i(\psi)}-\psi\left(y_{i(\psi)}\right) \rrbracket\right) /\left(y_{i(\psi)}-\psi\left(y_{i(\psi)}\right)\right),
$$

the $R$-module

$$
\widetilde{\mathrm{H}}^{\psi}(\underline{\hat{M}}, R):=\left\{\omega \in \mathrm{H}_{\mathrm{dR}}^{1}(\underline{\hat{M}}, R):[a]^{*} \omega=\psi(a) \cdot \omega \forall a \in \mathcal{O}_{E_{v}}\right\}
$$

was used (which was denoted without the $\sim$ on $\widetilde{\mathrm{H}}$ ) and in [2, Formula (1.13) and Definition 4.10]. Then $v\left(\omega_{\psi}\right)$ was defined to be

$$
v^{\sim}\left(\omega_{\psi}\right):=v(\tilde{x}) \in \mathbb{Q},
$$

where $\tilde{x} \in L^{\times}$is such that $\tilde{x}^{-1}\left(\omega_{\psi} \bmod y_{i(\psi)}-\psi\left(y_{i(\psi)}\right)\right)$ is an $R$-generator of $\widetilde{\mathrm{H}}^{\psi}(\underline{\hat{M}}, R)$. (To clarify the error we write $v^{\sim}\left(\omega_{\psi}\right)$ instead of $v\left(\omega_{\psi}\right)$ in this erratum.)

However, in the rest of [2] the $R$-submodule

$$
\mathrm{H}^{\psi}(\underline{\hat{M}}, R):=\mathrm{H}_{\mathrm{dR}}^{1}(\underline{\hat{M}}, R) /\left([a]^{*}-\psi(a): a \in \mathcal{O}_{E_{v}}\right) \cdot \mathrm{H}_{\mathrm{dR}}^{1}(\underline{\hat{M}}, R) \subset \mathrm{H}^{\psi}(\underline{\hat{M}}, L)
$$

is used as a reference integral structure on $\mathrm{H}^{\psi}(\underline{\hat{M}}, L)$. (See Lemma B.1 for why the latter is an inclusion and how the two integral structures can be compared.) Correspondingly, the following definition for $v\left(\omega_{\psi}\right)$ is used in [2]

$$
v\left(\omega_{\psi}\right):=v(x) \in \mathbb{Q}
$$

where $x \in L^{\times}$is such that $x^{-1}\left(\omega_{\psi} \bmod y_{i(\psi)}-\psi\left(y_{i(\psi)}\right)\right)$ is an $R$-generator of $\mathrm{H}^{\psi}(\underline{\hat{M}}, R)$. Indeed, in [2, Section 5.12] the generator $\omega_{\psi}^{\circ}:=1$ of $\mathrm{H}^{\psi}(\underline{\hat{M}}, R)$ is used, which might not lie in $\widetilde{\mathrm{H}}^{\psi}(\underline{\hat{M}}, R)$. Afterwards, any other generator $\omega_{\psi}$ is compared to the generator $\omega_{\psi}^{\circ}$. This error occurs in [2, Theorems 1.3 and 5.24 and Corollaries 5.22 and 5.25]. In terms of the valuation $v^{\sim}\left(\omega_{\psi}\right)$ from formula (B.2), all these theorems and corollaries have to be reformulated, as explained later. However, with the definition of $v\left(\omega_{\psi}\right)$ in formula (B.3), all these theorems and corollaries are correct.

Note that if $\underline{\hat{M}}=\underline{\hat{M}}_{v}(\underline{\mathcal{M}})$ arises from a good model $\underline{\mathcal{M}}$ of an $A$-motive over $R$ as in [2, Example 3.2] then $\mathrm{H}_{\mathrm{dR}}^{1}(\underline{\hat{M}}, R)=\mathrm{H}_{\mathrm{dR}}^{1}(\underline{\mathcal{M}}, R):=\sigma^{*} \mathcal{M} \otimes_{A_{R}, \gamma \otimes \mathrm{id}_{R}} R$, and hence

$$
\begin{aligned}
& \widetilde{\mathrm{H}}^{\psi}(\underline{\hat{M}}, R)=\widetilde{\mathrm{H}}^{\psi}(\underline{\mathcal{M}}, R):=\left\{\omega \in \mathrm{H}_{\mathrm{dR}}^{1}(\underline{\mathcal{M}}, R):[a]^{*} \omega=\psi(a) \cdot \omega \forall a \in \mathcal{O}_{E}\right\}, \\
& \mathrm{H}^{\psi}(\underline{\hat{M}}, R)=\mathrm{H}^{\psi}(\underline{\mathcal{M}}, R):=\mathrm{H}_{\mathrm{dR}}^{1}(\underline{\mathcal{M}}, R) /\left([a]^{*}-\psi(a): a \in \mathcal{O}_{E}\right) \cdot \mathrm{H}_{\mathrm{dR}}^{1}(\underline{\mathcal{M}}, R)
\end{aligned}
$$




$$
\text { inside } \quad \mathrm{H}^{\psi}(\underline{\hat{M}}, L)=\mathrm{H}^{\psi}(\underline{\mathcal{M}}, L)=\widetilde{\mathrm{H}}^{\psi}(\underline{\mathcal{M}}, R) \otimes_{R} L=\mathrm{H}^{\psi}(\underline{\mathcal{M}}, R) \otimes_{R} L .
$$

We next show how the two integral structures can be compared.

Lemma B.1. The integral structures $\widetilde{\mathrm{H}}^{\psi}(\underline{\hat{M}}, R)$ and $\mathrm{H}^{\psi}(\underline{\hat{M}}, R)$ are free $R$-modules of rank one and contained in the $L$-vector space $\mathrm{H}^{\psi}(\underline{\hat{M}}, L)$. The natural $R$-morphism

$$
\widetilde{\mathrm{H}}^{\psi}(\underline{\hat{M}}, R) \hookrightarrow \mathrm{H}_{\mathrm{dR}}^{1}(\underline{\hat{M}}, R) \longrightarrow \mathrm{H}^{\psi}(\underline{\hat{M}}, R)
$$

is injective with cokernel isomorphic to $R / R \cdot \psi\left(\mathfrak{D}_{E_{v} / Q_{v}}\right)$, where $\mathfrak{D}_{E_{v} / Q_{v}}$ is the different of $E_{v}=\prod_{i=1}^{s} E_{v, i}$ over $Q_{v}$.

Proof. The morphism fits into the diagram

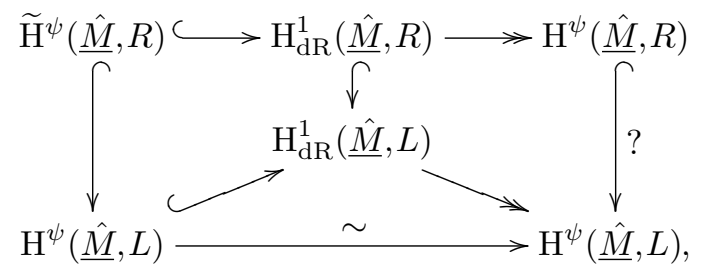

in which the lower isomorphism was described in formula (B.1), the lower triangle is the tensor product of the upper row with $L$ and the injectivity of the right vertical arrow still has to be proved. Note that the argument will not use the specific situation of de Rham cohomology of local shtukas. It will only use the isomorphism (B.1) coming from [2, Proposition 4.9] and the freeness of the $R$-module $\mathrm{H}_{\mathrm{dR}}^{1}(\underline{\hat{M}}, R)$ over $\mathcal{O}_{E, v} \otimes_{A_{v}} R$ (see later).

The $Q_{v}$-algebra $E_{v}$ acts on $\mathrm{H}^{\psi}(\underline{\hat{M}}, L)$ through the character $\psi: E_{v} \rightarrow E_{v, i(\psi)} \hookrightarrow L$. By $\left[3, \S I I I .6\right.$, Proposition 12], there exists an element $y \in \mathcal{O}_{E_{v, i(\psi)}}$ such that $\mathcal{O}_{E_{v, i(\psi)}}=A_{v}[y]=$ $A_{v}[Y] /(m)$, where $m \in A_{v}[Y]$ is the minimal polynomial of $y$ over $A_{v}$. The image $\gamma(m)$ under the map $\gamma: A_{v}[Y] \hookrightarrow R[Y]$ has $\psi(y)$ as a zero and correspondingly factors as

$$
\gamma(m)=(Y-\psi(y)) \cdot g(Y)
$$

for a monic polynomial $g(Y) \in R[Y]$. The derivative $m^{\prime}:=\frac{d m}{d Y} \in A_{v}[Y]$ satisfies

$$
\psi\left(m^{\prime}(y)\right)=\gamma(m)^{\prime}(\psi(y))=g(\psi(y)) .
$$

Recall that $A_{v, R}$ is the $v$-adic completion of $A_{R}$. By [2, Proposition 4.8] we can decompose $\underline{\hat{M}}=\bigoplus_{i=1}^{s} \underline{\hat{M}}_{i}$ into local shtukas $\underline{\hat{M}}_{i}$ over $R$ with complex multiplication by $\mathcal{O}_{E_{v, i}}$. In particular,

$$
\begin{aligned}
& \widetilde{\mathrm{H}}^{\psi}(\underline{\hat{M}}, R):=\left\{\omega \in \mathrm{H}_{\mathrm{dR}}^{1}\left(\underline{\hat{M}}_{i(\psi)}, R\right):[a]^{*} \omega=\psi(a) \cdot \omega \forall a \in \mathcal{O}_{E_{v, i}}\right\} \quad \text { and } \\
& \mathrm{H}^{\psi}(\underline{\hat{M}}, R):=\mathrm{H}_{\mathrm{dR}}^{1}\left(\underline{\hat{M}}_{i(\psi)}, R\right) /\left([a]^{*}-\psi(a): a \in \mathcal{O}_{E_{v, i}}\right) \cdot \mathrm{H}_{\mathrm{dR}}^{1}\left(\underline{\hat{M}}_{i(\psi)}, R\right)
\end{aligned}
$$

can be computed from

$$
\mathrm{H}_{\mathrm{dR}}^{1}\left(\underline{\hat{M}}_{i(\psi)}, R\right):=\sigma^{*} \hat{M}_{i(\psi)} \otimes_{A_{v, R}, \gamma \otimes \mathrm{id}_{R}} R
$$

instead of $\mathrm{H}_{\mathrm{dR}}^{1}(\underline{\hat{M}}, R)$. Moreover, by the same proposition $\underline{\hat{M}}$ is free over $\mathcal{O}_{E_{v}, R}:=\mathcal{O}_{E_{v}} \otimes_{A_{v}}$ $R \llbracket z \rrbracket=\mathcal{O}_{E_{v}} \widehat{\otimes}_{\mathbb{F}_{v}} R$ of rank one, and we may choose a generator of $\hat{M}$. This generator 
provides an isomorphism

$$
\mathrm{H}_{\mathrm{dR}}^{1}\left(\underline{\hat{M}}_{i(\psi)}, R\right) \cong\left(\mathcal{O}_{E_{v, i(\psi)}} \widehat{\otimes}_{\mathbb{F}_{q}} R\right) \underset{A_{v, R}, \gamma \otimes \mathrm{id}_{R}}{\otimes} R=\mathcal{O}_{E_{v, i(\psi)}} \underset{A_{v}, \gamma}{\otimes} R=R[Y] /(\gamma(m)) .
$$

Since $[a]^{*}-\psi(a)=[a]^{*}-\gamma(a)$ for $a \in A_{v}$ already annihilates $\mathrm{H}_{\mathrm{dR}}^{1}(\underline{\hat{M}}, R)$, this yields the upper vertical isomorphisms in the following diagram:

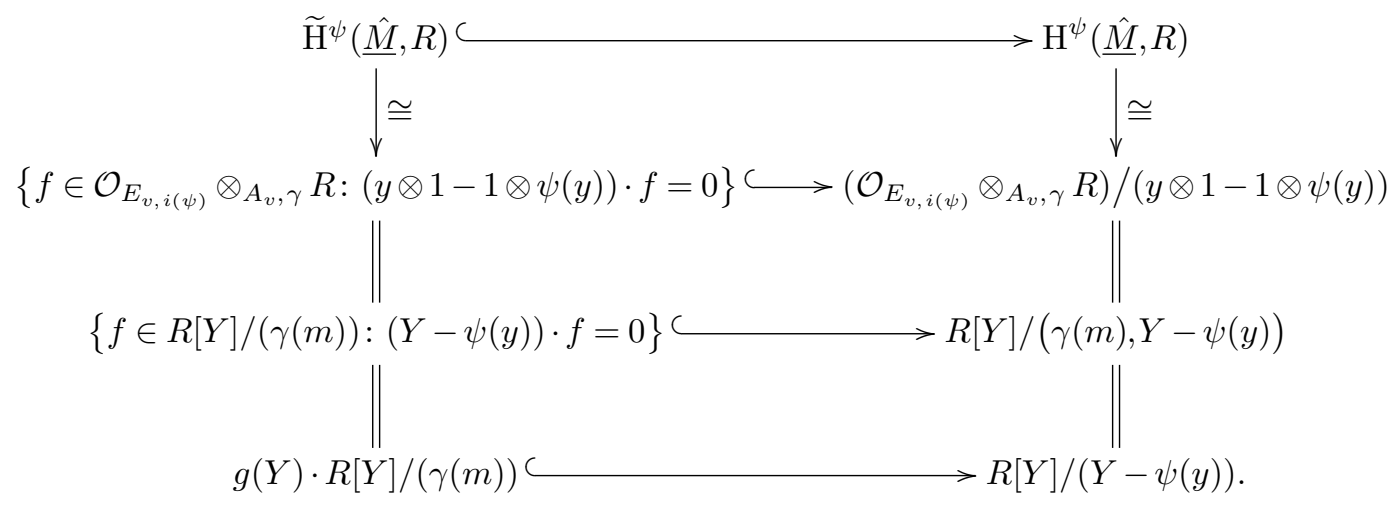

The injectivity of the horizontal maps follows from diagram (B.4). The lower left equality holds because $R[Y]$ has no $(Y-\psi(y))$-torsion. Next, $\mathrm{H}^{\psi}(\underline{\hat{M}}, R) \cong R[Y] /(Y-\psi(y)) \cong R$ is free, and hence contained in $\mathrm{H}^{\psi}(\underline{\hat{M}}, R) \otimes_{R} L=\mathrm{H}^{\psi}(\underline{\hat{M}}, L)$. Finally, the image of the lower horizontal map is the ideal

$$
R \cdot g(\psi(y))=R \cdot \psi\left(m^{\prime}(y)\right)=R \cdot \psi\left(\mathfrak{D}_{E_{v, i(\psi)} / Q_{v}}\right)=R \cdot \psi\left(\mathfrak{D}_{E_{v} / Q_{v}}\right)
$$

(see [3, §III.4, Proposition 10 and §III.6, Corollary 2].

Corollary B.2. For an L-generator $\omega_{\psi}$ of $\mathrm{H}^{\psi}(\underline{\hat{M}}, L)$, the two valuations in formulas (B.2) and (B.3) satisfy

$$
v\left(\omega_{\psi}\right)-v^{\sim}\left(\omega_{\psi}\right)=v\left(\mathfrak{D}_{\psi\left(E_{v}\right) / Q_{v}}\right)=v\left(\psi\left(\mathfrak{D}_{E_{v} / Q_{v}}\right)\right)=v\left(\psi\left(\mathfrak{D}_{E / Q}\right)\right) .
$$

Proof. Let $x, \tilde{x} \in L^{\times}$be elements such that $x^{-1} \omega_{\psi}$ is an $R$-generator of $\mathrm{H}^{\psi}(\underline{\hat{M}}, R)$ and $\tilde{x}^{-1} \omega_{\psi}$ is an $R$-generator of $\widetilde{\mathrm{H}}^{\psi}(\underline{\hat{M}}, R)$. Then $x / \tilde{x}$ is an $R$-generator of $\psi\left(\mathfrak{D}_{E_{v} / Q_{v}}\right)$ by Lemma B.1, and the corollary follows.

Now let $\underline{M}$ be an $A$-motive over a finite Galois extension $K \subset Q^{\text {alg }}$ of $Q$ with complex multiplication by a finite separable semi-simple $Q$-algebra $E$. Assume that $\psi(E) \subset K$ for all $\psi \in H_{E}$. Fix a $\psi \in H_{E}$ and let $\omega_{\psi}$ be a generator of the $K \llbracket y_{\psi}-\psi\left(y_{\psi}\right) \rrbracket$-module $\mathrm{H}^{\psi}\left(\underline{M}^{\eta}, K \llbracket y_{\psi}-\psi\left(y_{\psi}\right) \rrbracket\right)$. For every $\eta \in H_{K}$, let $\underline{M}^{\eta}$ and $\omega_{\psi}^{\eta} \in \mathrm{H}^{\eta \psi}\left(\underline{M}^{\eta}, K \llbracket y_{\eta \psi}-\eta \psi\left(y_{\eta \psi}\right) \rrbracket\right)$ be obtained by extension of scalars via $\eta$. With the corollary and the computation

$$
\begin{aligned}
\sum_{\eta \in H_{K}} v\left(\omega_{\psi}^{\eta}\right)-v^{\sim}\left(\omega_{\psi}^{\eta}\right) & =\sum_{\eta \in H_{K}} v\left(\eta \psi\left(\mathfrak{D}_{E / Q}\right)\right)=v\left(\prod_{\eta \in H_{K}} \eta \psi\left(\mathfrak{D}_{E / Q}\right)\right)=v\left(N_{K / Q}\left(\mathfrak{D}_{\psi(E) / Q}\right)\right) \\
& =v\left(N_{\psi(E) / Q}\left(N_{K / \psi(E)}\left(\mathfrak{D}_{\psi(E) / Q}\right)\right)\right)=[K: \psi(E)] \cdot v\left(\mathfrak{d}_{\psi\left(\mathcal{O}_{E}\right) / A}\right),
\end{aligned}
$$


we obtain a reformulation of [2, Theorems 1.3 and 5.24 and Corollaries 5.22 and 5.25] in terms of $v^{\sim}\left(\omega_{\psi}\right)$, which is even more analogous to [1, Theorem II.1.1(i)].

Theorem 1.3'. Let $\omega_{\psi}$ be a generator of the $K \llbracket y_{\psi}-\psi\left(y_{\psi}\right) \rrbracket$-module $\mathrm{H}^{\psi}\left(\underline{M}, K \llbracket y_{\psi}-\right.$ $\left.\psi\left(y_{\psi}\right) \rrbracket\right)$. For every $\eta \in H_{K}$, let $\underline{M}^{\eta}$ and $\omega_{\psi}^{\eta} \in \mathrm{H}^{\eta \psi}\left(\underline{M}^{\eta}, K \llbracket y_{\eta \psi}-\eta \psi\left(y_{\eta \psi}\right) \rrbracket\right)$ be obtained by extension of scalars via $\eta$, and choose an E-generator $u_{\eta} \in \mathrm{H}_{1, \operatorname{Betti}}\left(\underline{M}^{\eta}, Q\right)$. Then for every place $v \neq \infty$ of $C$, we have

$\frac{1}{\# H_{K}} \sum_{\eta \in H_{K}} v\left(\int_{u_{\eta}} \omega_{\psi}^{\eta}\right)=Z_{v}\left(a_{E, \psi, \Phi}^{0}, 1\right)-\mu_{\mathrm{Art}, v}\left(a_{E, \psi, \Phi}^{0}\right)+\frac{1}{\# H_{K}} \sum_{\eta \in H_{K}}\left(v^{\sim}\left(\omega_{\psi}^{\eta}\right)+v_{\eta \psi}\left(u_{\eta}\right)\right)$.

Corollary 5.22'. Let $\varphi, \psi \in H_{E_{v}}$ with $i(\varphi)=i(\psi)=: i$ and assume that $E_{v, i}$ is separable over $Q_{v}$. Let $u \in \mathrm{H}_{1, v}\left(\underline{\hat{M}}_{E_{v}, \varphi}, Q_{v}\right)$ be a generator as an $E_{v}$-module and let $\omega_{\psi}$ be an $L \llbracket y_{i}-$ $\psi\left(y_{i}\right) \rrbracket$-generator of $\mathrm{H}^{\psi}\left(\underline{\hat{M}}_{E_{v}, \varphi}, L \llbracket y_{i}-\psi\left(y_{i}\right) \rrbracket\right)$. Then $\int_{u} \omega_{\psi}:=u \otimes \operatorname{id}_{\mathbb{C}_{v}((z-\zeta))}\left(h_{v, \mathrm{dR}}^{-1}\left(\omega_{\psi}\right)\right)$ has valuation

$$
v\left(\int_{u} \omega_{\psi}\right)=Z_{v}\left(a_{E_{v}, \psi, \varphi}, 1\right)-\mu_{\mathrm{Art}, v}\left(a_{E_{v}, \psi, \varphi}\right)+v^{\sim}\left(\omega_{\psi}\right)+v_{\psi}(u)
$$

where $v^{\sim}\left(\omega_{\psi}\right)$ and $v_{\psi}(u)$ are defined in forumla (B.2) and [2, Definition 5.21] respectively.

Theorem 5.24'. Let $\underline{\hat{M}}$ be a local shtuka over $R$ with complex multiplication by the ring of integers $\mathcal{O}_{E_{v}}$ in a commutative, semi-simple, separable $Q_{v}$-algebra $E_{v}$ with local $C M$ type $\Phi$, and assume that $\psi\left(E_{v}\right) \subset L$ for all $\psi \in H_{E_{v}}$ and that $L$ is separable over $Q_{v}$. Let $u \in \mathrm{H}_{1, v}\left(\underline{\hat{M}}, Q_{v}\right)$ be an $E_{v}$-generator and let $\omega_{\psi}$ be an $L \llbracket y_{i(\psi)}-\psi\left(y_{i(\psi)}\right) \rrbracket$-generator of $\mathrm{H}^{\psi}\left(\underline{\hat{M}}, L \llbracket y_{i(\psi)}-\psi\left(y_{i(\psi)}\right) \rrbracket\right)$. Then the period $\int_{u} \omega_{\psi}:=u \otimes \operatorname{id}_{\mathbb{C}_{v}((z-\zeta))}\left(h_{v, \mathrm{dR}}^{-1}\left(\omega_{\psi}\right)\right)$ has valuation

$$
v\left(\int_{u} \omega_{\psi}\right)=Z_{v}\left(a_{E_{v}, \psi, \Phi}, 1\right)-\mu_{\mathrm{Art}, v}\left(a_{E_{v}, \psi, \Phi}\right)+v^{\sim}\left(\omega_{\psi}\right)+v_{\psi}(u)
$$

where $v^{\sim}\left(\omega_{\psi}\right)$ and $v_{\psi}(u)$ are defined in formula (B.2) and [2, Definition 5.21] respectively.

Corollary 5.25'. Keep the situation of Theorem 5.24'. For every $\eta \in H_{L}$, note that $i(\eta \psi)=i(\psi)$, let $\underline{\hat{M}}^{\eta}$ and $\omega_{\psi}^{\eta} \in \mathrm{H}^{\eta \psi}\left(\underline{\hat{M}}^{\eta}, L \llbracket y_{i(\psi)}-\eta \psi\left(y_{i(\psi)}\right) \rrbracket\right)$ be obtained by extension of scalars via $\eta$ and choose an $E_{v}$-generator $u_{\eta} \in \mathrm{H}_{1, v}\left(\underline{\hat{M}}^{\eta}, Q_{v}\right)$. Then

$\frac{1}{\# H_{L}} \sum_{\eta \in H_{L}} v\left(\int_{u_{\eta}} \omega_{\psi}^{\eta}\right)=Z_{v}\left(a_{E_{v}, \psi, \Phi}^{0}, 1\right)-\mu_{\mathrm{Art}, v}\left(a_{E_{v}, \psi, \Phi}^{0}\right)+\frac{1}{\# H_{L}} \sum_{\eta \in H_{L}}\left(v^{\sim}\left(\omega_{\psi}^{\eta}\right)+v_{\eta \psi}\left(u_{\eta}\right)\right)$.

\section{References}

[1] P. Colmez, Périodes des variétés abéliennes a multiplication complexe, Ann. of Math. (2) 138(3) (1993), 625-683.

[2] U. Hartl and R. K. Singh, Periods of Drinfeld modules and local shtukas with complex multiplication, J. Inst. Math. Jussieu 19(1) (2020), 175-208.

[3] J.-P. SERre, Local Fields, Graduate Texts in Mathematics, 67 (Springer-Verlag, New York, 1979). 